\title{
A comparison of bone mineral density in adolescent swimmers, pentathletes and figure skaters
}

\author{
Ersin Gümüş ${ }^{1}$, Sinem Akgül'2, Nuray Kanbur², Orhan Derman ${ }^{2}$ \\ ${ }^{1}$ Department of Pediatric Gastroenterology, Balıkesir Atatürk City Hospital, Balıesir; ${ }^{2}$ Division of \\ Adolescent Medicine, Department of Pediatrics, Hacettepe University Faculty of Medicine, Ankara, Turkey. \\ E-mail:ersin.gumus@hacettepe.edu.tr \\ Received: 18th December 2019, Revised: 23rd December 2019, Accepted: 23rd December 2019
}

SUMMARY: Gümüş E, Akgül S, Kanbur N, Derman O. A comparison of bone mineral density in adolescent swimmers, pentathletes and figure skaters. Turk J Pediatr 2019; 61: 831-838.

Physical activity accounts for up to $17 \%$ of the variance in bone mineral density (BMD). Specifically, weight bearing exercises are of critical importance in increasing BMD. The aim of the present study was to evaluate the influence of three different sports with different mechanical loading properties on BMD of young athletes. This was an observational retrospective cohort study. All available medical records of adolescent athletes who have been followed at the Division of Adolescent Medicine at Hacettepe University were reviewed. Bone mineral content (BMC) and BMD of lumbar spine (L1-L4) and left femoral region were compared between young athletes participating in swimming $(n=50)$, pentathlon $(n=18)$, and figure skating $(n=7)$. Femur total and lumbar spine (L1-L4) BMC values of figure skaters $(29.1 \pm 8.1 \mathrm{~g}$ and $51.5 \pm 19.0 \mathrm{~g}$, respectively) were found to be higher than swimmers $(21.5 \pm 6.6 \mathrm{~g}$ and $36.1 \pm 11.7 \mathrm{~g}$, respectively) and pentathletes $(21.6 \pm 6.2 \mathrm{~g}$ and $41.7 \pm 19.4 \mathrm{~g}$, respectively). However, there were no significant differences between any of the groups in lumbar spine and left proximal femur measurements. Regarding $\mathrm{BMD}$, figure skaters $\left(1.1 \pm 0.2 \mathrm{~g} / \mathrm{cm}^{2}\right)$ had significantly higher femur total BMD values than pentathletes $\left(0.9 \pm 0.1 \mathrm{~g} / \mathrm{cm}^{2}\right)$ and swimmers $\left(0.9 \pm 0.1 \mathrm{~g} / \mathrm{cm}^{2}, p\right.$ $<0.05)$. The type of sport has an impact on BMD. Weight bearing and high impact loading activities seem to be associated with a better bone density in a region specific manner. Recommendation of high impact loading exercises may be considered to improve bone health in young athletes especially participating in low impact and non-weight bearing sports.

Key words: adolescent, bone mineral density, high impact loading, weight bearing.

Osteoporosis is a systemic skeletal disease characterized by reduced amount of bone mass and micro-structural deterioration, and associated with an increase in bone fragility and susceptibility to fractures. ${ }^{1}$ Peak bone mass (PBM) is a major determinant of bone mass later in life and about $90 \%$ of peak bone mass is reached by the end of the second decade in both sexes. ${ }^{2}$ Environmental factors such as hormones, dietary intake, calcium and vitamin $\mathrm{D}$ intake, medication use, sedentary lifestyle, smoking, alcohol use, and physical activity are thought to be responsible for the $\mathrm{PBM}$ variance. ${ }^{3}$ Physical activity is one of the most important factors that affect bone density and geometry. Physical activity accounts for up to $17 \%$ of the variance in bone mineral density (BMD). ${ }^{4}$ The skeleton adapts to the loading that results from specific sports activities and changes its structure and mass in response to dynamic loads. ${ }^{5}$ Different forms of exercise can cause different mechanical loadings. 
Specifically, weight bearing exercises are of critical importance in increasing BMD. ${ }^{6}$

Dual-energy X-ray absorptiometry (DXA) provides measures of bone mineral content (BMC) and BMD that are used to assess bone health. Increased BMC and BMD suggest increased bone strength and greater resistance to fracture. DXA is the most commonly used bone densitometric technique for children throughout the world and preffered over other techniques because of its speed, precision, safety, low cost, and widespread availability. ${ }^{7}$

Swimming represents a non-weight bearing sport that affects bone metabolism by increased muscle contraction and strain on the skeleton, hence leading to increased mechanical loading. ${ }^{8}$ Although swimming provides an active loading to skeletal structures, the absence of intermittent highimpact ground-reaction forces and presence of hypogravity during swimming seem to be responsible for the lower BMD and BMC values when compared to high or odd impact sports. ${ }^{9}$ In most of the studies swimmers had similar bone mineral density values compared to sedentary controls. ${ }^{10-13}$

Figure skating is a sport that consists of repetitive, high impact, bone loading activities, which favor bone accretion. It has already been shown that figure skaters have $15-24 \%$ higher calcaneal BMD values than controls. ${ }^{14}$ The osteogenic effect of figure skating was found to be most apparent in single skaters whose training includes more jumps than other figure skating disciplines, emphasizing the importance of landing forces and mechanical bone loading on bone accretion. ${ }^{15}$

The modern pentathlon is an Olympic event that comprises pistol shooting, fencing, horse riding, swimming, and running events. The pentathlon is a multidiscipline sport with unique mechanical loading pattern that incorporates both weight bearing and nonweight bearing conditions. To the best of our knowledge there hasn't been any study evaluating the effect of participating in pentathlon on BMD.

The purpose of our study was to evaluate the influence of different sports with different mechanical loading properties (weight bearing, non-weight bearing and combinations of both types of loading patterns) on BMD among young athletes.

\section{Material and Methods}

\section{Participants}

This was an observational retrospective cohort study. All available medical records of adolescent athletes who have been followed at the Division of Adolescent Medicine at Hacettepe University were reviewed and relevant clinical data and results of BMC/ BMD measurements were collected. Seventyfive healthy subjects (age range 10-18 years) were enrolled for the study. The study sample included three groups of subjects participating in different sports: swimming $(n=50)$, pentathlon $(n=18)$, and figure skating $(n=7)$. Subjects were included in the study only if they had been involved in their respective sport for at least two years, trained regularly at least three days per week and a minimum of 10 months per year, and were not currently involved in any other organized sport or any form of weight bearing exercise. Included subjects were free of any chronic disease and of any medication that would affect bone and muscle metabolism. Results of routine laboratory tests, left-hand wrist radiograph for determining bone age and BMC/BMD of lumbar spine and left femoral region for evaluating bone health were recorded for every participant. Data regarding physical and sexual maturation of adolescent athletes were obtained from examination charts. All participants were evaluated individually for staging sexual maturation by using Tanner's classification.

This study was approved by the Institutional Ethics Committee on Non-Interventional Clinical Research (GO14/110-05) and carried out according to the Declaration of Helsinki protocol. Written informed consent was obtained from all individual participants and their parents for participation in the study.

\section{Evaluation of diet}

Dietary information, obtained from food diaries was recorded when available. Daily 
calorie and calcium intakes of subjects with available dietary information were calculated from food dairies by a dietitian.

\section{Anthropometric measurements}

Anthropometric measurements (body weight and height) were taken using conventional procedures. Body weight $(\mathrm{kg})$ was measured with an electric scale and body height $(\mathrm{cm})$ with a height meter. The body mass index (BMI) was calculated by dividing the body mass by the square of height $\left(\mathrm{kg} \cdot \mathrm{m}^{-2}\right)$. Greulich and Pyle's Radiographic Atlas of Skeletal Development of the Hand and Wrist was used for determination of bone age.

\section{Evaluation of bone mineral density}

Dual-energy x-ray absorptiometry technique (DXA-Lunar Prodigy, GE Medical Systems, USA) was used for the measurement of regional bone mineral density (BMD, g/ $\mathrm{cm}^{2}$ ) and bone mineral content (BMC, g) at the lumbar spine (L1-L4) and left proximal femoral region (femoral neck, Ward triangle and greater trochanter).

\section{Statistics}

The data were analyzed using SPSS (version 16.0 for Windows; SPSS Inc, Chicago, IL). The statistical comparisons for the different variables among the groups were performed by applying a one-way analysis of variance (ANOVA) or Kruskal Wallis test with regard to parametric test assumptions were met or not. Tukey post hoc testing was performed when necessary. Comparisons between sports groups were completed using a univariate analysis of covariance (ANCOVA) with body mass index (BMI) and age as covariates because of the known relationship between body weight, height, age, and bone density. Level of significance was set at $\mathrm{p}<0.05$.

\section{Results}

\section{Descriptive characteristics}

Descriptive characteristics of subjects for each sport group are summarized in Table I. Sport groups were matched on gender and Tanner stage. Despite the fact that figure skaters were older than the swimmers, bone age was similar among groups. There were no significant differences between sport groups for weight and height. Pentathlon group had significantly lower BMI values than swimmers. Figure skaters had been involved in specialized training significantly longer than pentathletes and swimmers. No differences between groups were found for daily calorie and calcium intake. Overall mean daily calorie and calcium intake of all included subjects were $1894.9 \pm$ $464 \mathrm{kcal}$ and $616.4 \pm 211.2 \mathrm{mg}$, respectively.

Table I. Descriptive Characteristics by Sport Type.

\begin{tabular}{|c|c|c|c|c|}
\hline \multirow[b]{2}{*}{ Characteristics } & \multicolumn{3}{|c|}{ Sport Type } & \multirow[b]{2}{*}{$p$} \\
\hline & $\begin{array}{l}\text { Swimming } \\
\text { (n: 50) }\end{array}$ & $\begin{array}{l}\text { Pentathlon } \\
\text { (n: 18) }\end{array}$ & $\begin{array}{l}\text { Figure skating } \\
\text { (n: } 7)\end{array}$ & \\
\hline Female/male, $\mathrm{n} / \mathrm{n}$ & $23 / 27$ & $9 / 9$ & $4 / 3$ & \\
\hline Age, years & $11.8 \pm 1.6$ & $13 \pm 2.5$ & $14.3 \pm 2.1$ & $<0.01$ \\
\hline Weight, kg & $45.1 \pm 10.8$ & $41.0 \pm 10.7$ & $48.1 \pm 11.3$ & 0.40 \\
\hline Height, $\mathrm{cm}$ & $152.0 \pm 11.5$ & $154.3 \pm 13.1$ & $158.3 \pm 14.6$ & 0.25 \\
\hline BMI, kg.m ${ }^{-2}$ & $19.2 \pm 2.3$ & $16.9 \pm 2.2$ & $18.9 \pm 1.8$ & $<0.05$ \\
\hline Bone age, years & $11.7 \pm 1.9$ & $12.9 \pm 2.8$ & $13.7 \pm 2.5$ & 0.08 \\
\hline Height age, years & $12.7 \pm 2.5$ & $13.0 \pm 2.7$ & $14.9 \pm 3.6$ & 0.11 \\
\hline Months in sports & $54.4 \pm 22.2$ & $30.0 \pm 15.8$ & $73.7 \pm 40.6$ & $<0.001$ \\
\hline \multicolumn{5}{|c|}{ Pubertal stage, n (\%) } \\
\hline Stage 1-3 & $42(84)$ & $13(72)$ & $6(87)$ & \\
\hline Stage 4-5 & $8(16)$ & $5(28)$ & $1(13)$ & \\
\hline
\end{tabular}

BMI: body mass index. Results are shown as mean \pm standard deviation. 


\section{$B M C$ and $B M D$}

The results of bone densitometry are shown in Table II and Table III. Although BMC values of figure skaters were found to be higher than other two groups, there were no significant differences between any of the groups in lumbar spine and left proximal femur. Regarding BMD, there were no significant differences between any of the groups in lumbar spine. However, figure skaters had significantly higher BMD values than pentathletes and swimmers for femur total, femoral neck and trochanter regions $(p<0.05)$.

\section{Laboratory values}

The results of routine laboratory tests are summarized in Table IV. There were no significant differences between groups for biochemical parameters related to bone health including hemoglobin, serum calcium, phosphorus, alkaline phosphatase. When compared to swimmers $(26.7 \pm 13.3 \mathrm{ng} / \mathrm{ml})$ and figure skaters $(30.8 \pm 20.5 \mathrm{ng} / \mathrm{ml})$ serum ferritin levels of pentathletes $(15.8 \pm 10.9 \mathrm{ng} /$ $\mathrm{ml})$ were significantly lower $(p<0.05)$. Serum levels of osteocalcin, a specific bone formation marker, were significantly higher in swimming group $(p<0.05)$. Although serum 25 -hydroxy vitamin D levels were not significantly different between groups, figure skaters tended to have lower values. When all subjects taken together, $43 \%$ of the athletes found to have vitamin D deficiency which is defined as values below 20 $\mathrm{ng} / \mathrm{ml}$. No significant correlation was observed between BMD/BMC values and biochemical data in the overall group of athletes.

\section{Discussion}

This study evaluated the potential differences in BMC and BMD of adolescents participating in different sports with different mechanical loading properties. The main finding of this study was that figure skaters had significantly higher BMD values than pentathletes and

Table II. Bone Mineral Content Values of Lumbar and Femoral Regions by Sport Type.

\begin{tabular}{lcccc}
\hline \multirow{2}{*}{ Regions } & \multicolumn{3}{c}{ Sport Type } & \\
\cline { 2 - 3 } & $\begin{array}{c}\text { Swimming } \\
\text { (n: 50) }\end{array}$ & $\begin{array}{c}\text { Pentathlon } \\
\text { (n: 18) }\end{array}$ & $\begin{array}{c}\text { Figure skating } \\
\text { (n: 7) }\end{array}$ & $p$ \\
\hline Femur BMC, g & & & & \\
$\quad$ Neck & $3.7 \pm 1.0$ & $3.7 \pm 0.9$ & $4.8 \pm 1.3$ & 0.19 \\
$\quad$ Wards & $1.8 \pm 0.7$ & $1.7 \pm 0.5$ & $2.5 \pm 0.9$ & 0.27 \\
$\quad$ Trochanter & $5.6 \pm 2.5$ & $6.0 \pm 2.3$ & $8.6 \pm 3.3$ & 0.33 \\
$\quad$ Total & $21.5 \pm 6.6$ & $21.6 \pm 6.2$ & $29.1 \pm 8.1$ & 0.26 \\
Lumbar spine BMC (L1-L4), g & $36.1 \pm 11.7$ & $41.7 \pm 19.4$ & $51.5 \pm 19.0$ & 0.36 \\
\hline
\end{tabular}

BMC: bone mineral content (gram). Results are shown as mean \pm standard deviation.

Table III. Bone Mineral Density Values of Lumbar and Femoral Regions by Sport Type.

\begin{tabular}{lcccc}
\hline \multirow{2}{*}{ Regions } & \multicolumn{3}{c}{ Sport Type } & \multirow{2}{*}{$p$} \\
\cline { 2 - 3 } & $\begin{array}{c}\text { Swimming } \\
\text { (n: 50) }\end{array}$ & $\begin{array}{c}\text { Pentathlon } \\
\text { (n: 18) }\end{array}$ & $\begin{array}{c}\text { Figure skating } \\
\text { (n: 7) }\end{array}$ & \\
\hline Femur BMD, g/cm $\mathrm{cm}^{2}$ & & & & \\
$\quad$ Neck & $0.9 \pm 0.1$ & $0.9 \pm 0.1$ & $1.0 \pm 0.2$ & $<0.05$ \\
$\quad$ Wards & $0.8 \pm 0.1$ & $0.9 \pm 0.1$ & $1.0 \pm 0.2$ & 0.13 \\
$\quad$ Trochanter & $0.7 \pm 0.1$ & $0.7 \pm 0.1$ & $0.9 \pm 0.2$ & $<0.05$ \\
$\quad$ Total & $0.9 \pm 0.1$ & $0.9 \pm 0.1$ & $1.1 \pm 0.2$ & $<0.05$ \\
Lumbar spine BMD (L1-L4), g/cm $\mathrm{cm}^{2}$ & $0.9 \pm 0.2$ & $0.9 \pm 0.2$ & $1.1 \pm 0.2$ & 0.83 \\
\hline
\end{tabular}

BMD: bone mineral density. Results are shown as mean \pm standard deviation. 
Table IV. Laboratory Findings Related to Bone Health by Sport Type.

\begin{tabular}{lcccc}
\hline \multirow{2}{*}{ Biochemical parameters } & \multicolumn{3}{c}{ Sport Type } & \multirow{2}{*}{$p$} \\
\cline { 2 - 4 } & Swimming & Pentathlon & Figure skating & \\
\hline Hemoglobin, g/dl & $13.8 \pm 0.81$ & $13.3 \pm 1.19$ & $13.4 \pm 1.35$ & 0.14 \\
Ferritin, ng/ml & $26.7 \pm 13.3$ & $15.8 \pm 10.9$ & $30.8 \pm 20.5$ & $<0.05$ \\
Serum calcium, mg/dl & $9.8 \pm 0.29$ & $9.9 \pm 0.33$ & $9.7 \pm 0.39$ & 0.65 \\
Serum phosphorus, mg/dl & $4.3 \pm 0.51$ & $4.4 \pm 0.59$ & $4.4 \pm 0.65$ & 0.88 \\
ALP, U/ml & $199.2 \pm 63.52$ & $214.4 \pm 77.25$ & $214.7 \pm 61.8$ & 0.66 \\
Osteocalcin, ng/ml & $58.0 \pm 32.3$ & $22.4 \pm 21.8$ & $30.8 \pm 19.13$ & $<0.05$ \\
25-(OH)D, ng/ml & $25.2 \pm 12.26$ & $25.1 \pm 9.33$ & $15.0 \pm 8.13$ & 0.13 \\
\hline
\end{tabular}

ALP: alkaline phosphatase, 25-(OH)D: 25-hydroxy vitamin D. Results are shown as mean \pm standard deviation.

swimmers in a region specific manner. Our finding is in agreement with the previous literature reporting lower lumbar spine and limb BMD values in participants of nonweight-bearing activities compared to weightbearing activities. ${ }^{12}$

Swimming represents a repetitive, nonweight bearing sport without direct impact loading to skeletal structures. In our study, swimmers had the lowest BMD and BMC values in both lumbar spine and femoral region compared to pentathletes and figure skaters. Many studies with similar results have been reported previously. Swimmers were found to have similar BMD and/or BMC values when compared to sedentary controls and lower BMD values than any other sport group independently of the age of the sample. ${ }^{13}$ Relative weightlessness and lack of daily impact due to training in a hypogravity environment can be held responsible for negative influence on bone mineral acquisition in swimming. On the other hand, in our study, osteocalcin which is a specific bone formation marker was found to be higher in swimmers than pentathletes and figure skaters. Several studies have compared bone formation markers including bone-specific alkaline phosphatase and osteocalcin between swimming and other sport groups. Most of the studies revealed that swimmers had similar ostecalcin levels when compared to controls or sport groups. ${ }^{16-19}$ In only one study, serum osteocalcin levels of swimmers were found to be higher than controls but similar with other sport groups. ${ }^{20}$ Higher osteocalcin levels of swimmers in our study could be related to a high bone turnover rate which may be associated with a more efficient bone structure without producing any differences in BMD.

To the best of our knowledge this is the first study to report BMD values of pentathletes and compare them with other sports. Pentathlon is a multidiscipline sport consisting of pistol shooting, fencing, horse riding, swimming and running events with unique mechanical loading patterns. While running and fencing are weight bearing activities with ground reaction forces, swimming, pistol shooting and horse riding can be considered as predominantly non-weight bearing activities with no or little ground reaction forces. As pentathlon incorporates both weight bearing and non-weight bearing activities together we expected that pentathlon would have a positive impact on BMD of young athletes. However, our results showed that pentathletes have similar bone density values as swimmers and lower left femur BMD measurements than figure skaters. Despite the lack of information about the impact of pentathlon on BMD of athletes, there have been some reports regarding triathlon which is also a multidiscipline sport consisting of running, cycling and swimming. In the literature, athletes participating in triathlon were found to have significantly higher adjusted leg BMD and a non-significant trend toward higher femoral neck BMD compared to swimmers suggesting a positive osteogenic influence of regional weight bearing loading exercise. ${ }^{12,21}$ The reasons for absence of positive effects of weight bearing activities and unusual strain patterns involved in pentathlon on bone 
acquisition can be distinct. It can be concluded that the weightlessness of swimming might attenuate the expected positive benefits of running. Also, pentathletes tend to devote most of their practice time for swimming. In addition, leanness which is promoted in pentathlon may result in attenuation of positive effects of body weight on bone mineral acquisition. In line with this, our pentathlon group had significantly lower BMI values. There is also evidence that high volumes of running, cycling or swimming can have a negative impact on bone mineral density. ${ }^{22,23}$ Pentathletes usually engage in high volumes of swimming and running trainings which may partly be responsible for absence of expected bone accretion.

Figure skating training mainly consists of jumps which are in nature repetitive, high impact, and bone loading activities. Also, figure skating is a sport in which leanness is especially preferred for performance and aesthetic concerns. Leanness may have negative effects on bone health especially in combination with calorie restriction and intense physical activity. There is limited data regarding effect of figure skating on bone density. And this study is the first one, to our knowledge, comparing figure skaters with different bone loading types of sports. The landing foot of skaters without a fracture history was found to have a significantly greater estimated BMD than the takeoff foot for jumping. ${ }^{14}$ It has also been shown that bone mineral density of skaters varies across discipline. Single skaters whose short program and training include more jumps found to have greater BMD in the regions receiving ground reaction forces such as pelvic and leg regions. ${ }^{15}$ In a study which compared young female figure skaters with non-athletic control subjects to ascertain whether there were differences in skeletal densities at various sites, similar skeletal densities at upper body sites (spine, arms, ribs) and significantly greater densities in the pelvis and legs were found. ${ }^{24}$ In consistence with literature findings figure skaters in our study group had significantly higher femoral BMD values than pentathletes and swimmers. Since the age of figure skaters were higher than that of swimmers and pentathletes, involving in specialized training significantly longer than the other two groups may have also contributed to higher BMD values in this group.

Nutrition has been shown to be an important environmental factor affecting PBM during childhood and adolescence. ${ }^{25}$ Processes involved in bone health including bone tissue deposition, maintenance and repair require adequate intake of energy, macronutrients and micronutrients. Calcium is one of the most important nutritional factors affecting bone mineralization especially before puberty. ${ }^{26}$ Increasing calcium intake may contribute to bone mass accrual in adolescents and decrease the risk for osteoporosis and fractures. ${ }^{27}$ Protein-energy malnutrition can also lead to decreased formation of cortical bone. ${ }^{5}$ Daily calorie and calcium intakes below the recommended values in our study group underscores the importance of closely assessing the nutritional status of these competitive young athletes and taking preemptive measures against complications of decreased bone density. Although we did not find any association between low serum 25-hydroxy vitamin D levels and BMC/ $\mathrm{BMD}$ values, vitamin $\mathrm{D}$ has been reported to be associated with accrual of bone mineral density in different studies. ${ }^{28-29}$ Lower values of vitamin $\mathrm{D}$ in figure skaters in our study may be multifactorial and related to inadequate exposure to sunlight due to inside practices, low dietary intake or the seasonal timing of blood sampling from subjects. In a recent study, a positive association between serum ferritin levels and BMC in healthy adolescent boys has been reported with a suggestion of monitoring iron stores to improve bone health. ${ }^{30}$ Ferritin levels of pentathletes in the present study were not only significantly lower than the other two sport groups, but also below the target threshold $(20 \mathrm{ng} / \mathrm{ml})$ suggested for competitive athletes. ${ }^{31}$ Although no significant correlation was observed between BMD/BMC values and biochemical data in the overall group of athletes, important nutritional deficiencies including inadequate energy and calcium intake, hypovitaminosis $\mathrm{D}$ and low iron stores are important problems among young athletes necessitating proper supplementation. 
There are limitations of our study. As gender and pubertal stage have an effect on bone mineral density it would have been better if analyses were performed in a gender and puberty specific manner. Sport groups in our study were matched for gender. However, the relatively small sample size of figure skaters limited the statistical power and did not allow subgroup analyses. A control group was not available and we could not evaluate if there was a significant difference regarding BMD between each sport group and healthy subjects not participating in sports. We only used BMD and $\mathrm{BMC}$ values for assessing the impact of different sport types on bone health. However, bone structure and geometry also have great importance in skeletal strength and should be evaluated.

The findings of this study show that the type of sport has an impact on BMD. Weight bearing and high impact loading activities seem to be associated with a better bone density in a region specific manner. Implementation of high impact loading exercises in the training of young athletes who participate in a sport with low weight bearing properties may have a positive impact on bone mineral density which is a good indicator of future bone health.

\section{REFERENCES}

1. NIH Consensus Development Panel on Osteoporosis Prevention, Diagnosis, and Therapy, March 7-29, 2000: Highlights of the conference. South Med J 2001; 94: 569-573.

2. Baxter-Jones ADG, Faulkner RA, Forwood MR, Mirwald RL, Bailey DA. Bone mineral accrual from 8 to 30 years of age: an estimation of peak bone mass. J Bone Miner Res 2011; 26: 1729-1739.

3. Ishikawa S, Kim Y, Kang M, Morgan DW. Effects of weight-bearing exercise on bone health in girls: a meta-analysis. Sports Med 2013; 43: 875-892.

4. Welten DC, Kemper HC, Post GB, et al. Weightbearing activity during youth is a more important factor for peak bone mass than calcium intake. J Bone Miner Res 1994; 9: 1089-1096.

5. Heaney RP, Abrams S, Dawson-Hughes B, et al. Peak bone mass. Osteoporos Int 2000; 11: 985-1009.

6. Asikainen TM, Kukkonen-Harjula K, Miilunpalo S. Exercise for health for early postmenopausal women: a systematic review of randomised controlled trials. Sports Med 2004; 34: 753-778.
7. Gordon CM, Bachrach LK, Carpenter TO, et al. Dual energy X-ray absorptiometry interpretation and reporting in children and adolescents: the 2007 ISCD Pediatric Official Positions. J Clin Densitom 2008; 11: 43-58.

8. Akgul S, Kanbur N, Cinemre SA, Karabulut E, Derman O. The effect of swimming and type of stroke on bone metabolism in competitive adolescent swimmers: a pilot study. Turk J Med Sci 2015; 45: 827-832.

9. Tenforde AS, Fredericson M. Influence of sports participation on bone health in the young athlete: a review of the literature. PM R 2011; 3: 861-867.

10. Bellew JW, Gehrig L. A comparison of bone mineral density in adolescent female swimmers, soccer players, and weight lifters. Pediatr Phys Ther 2006; 18: 19-22.

11. Courteix D, Lespessailles E, Peres SL, Obert P, Germain P, Benhamou CL. Effect of physical training on bone mineral density in prepubertal girls: a comparative study between impact-loading and nonimpact-loading sports. Osteoporos Int 1998; 8: 152158.

12. Duncan CS, Blimkie CJR, Cowell CT, Burke ST, Briody JN, Howman-Giles R. Bone mineral density in adolescent female athletes: relationship to exercise type and muscle strength. Med Sci Sports Exerc 2002; 34: 286-294.

13. Gomez-Bruton A, Gonzalez-Aguero A, GomezCabello A, Casajus JA, Vicente-Rodriguez G. Is bone tissue really affected by swimming? A systematic review. PLoS One 2013; 8: e70119.

14. Oleson CV, Busconi BD, Baran DT. Bone density in competitive figure skaters. Arch Phys Med Rehabil 2002; 83: 122-128.

15. Prelack K, Dwyer J, Ziegler P, Kehayias JJ. Bone mineral density in elite adolescent female figure skaters. J Int Soc Sports Nutr 2012; 9: 57.

16. Derman O, Cinemre A, Kanbur N, Dogan M, Kilic M, Karaduman E. Effect of swimming on bone metabolism in adolescents. Turk J Pediatr 2008; 50: 149-154.

17. Maimoun L, Coste $\mathrm{O}$, Philibert $\mathrm{P}$, et al. Testosterone secretion in elite adolescent swimmers does not modify bone mass acquisition: a 1-year follow-up study. Fertil Steril 2013; 99: 270-278.

18. Maimoun L, Coste O, Philibert P, et al. Peripubertal female athletes in high-impact sports show improved bone mass acquisition and bone geometry. Metabolism 2013; 62: 1088-1098.

19. Morgan A, Weiss Jarrett J. Markers of bone turnover across a competitive season in female athletes: a preliminary investigation. J Sports Med Phys Fitness 2011; 51: 515-524. 
20. Maimoun L, Mariano-Goulart D, Couret I, et al. Effects of physical activities that induce moderate external loading on bone metabolism in male athletes. J Sports Sci 2004; 22: 875-883.

21. Woodhead HJ, Kemp AF, Blimkie CJR, et al. Measurement of midfemoral shaft geometry: repeatability and accuracy using magnetic resonance imaging and dual-energy X-ray absorptiometry. J Bone Miner Res 2001; 16: 2251-2259.

22. Barrack MT, Rauh MJ, Nichols JF. Cross-sectional evidence of suppressed bone mineral accrual among female adolescent runners. J Bone Miner Res 2010; 25: 1850-1857.

23. Smathers AM, Bemben MG, Bemben DA. Bone density comparisons in male competitive road cyclists and untrained controls. Med Sci Sports Exerc 2009; 41: 290-296.

24. Slemenda CW, Johnston CC. High intensity activities in young women: site specific bone mass effects among female figure skaters. Bone Miner 1993; 20: 125-132.

25. Dogan M, Derman O, Oksuz-Kanbur N, Akgul S, Kutluk T. The effects of nutrition and physical activity on bone development in male adolescents. Turk J Pediatr 2009; 51: 545-550.
26. Johnston CCJr, Miller JZ, Slemenda CW, et al. Calcium supplementation and increases in bone mineral density in children. N Engl J Med 1992; 327: 82-87.

27. Ma XM, Huang ZW, Yang XG, Su YX. Calcium supplementation and bone mineral accretion in Chinese adolescents aged 12-14 years: a 12-month, dose-response, randomised intervention trial. $\mathrm{Br} \mathrm{J}$ Nutr 2014; 112: 1510-1520.

28. Lehtonen-Veromaa MKM, Mottonen TT, Nuotio IO, Irjala KMA, Leino AE, Viikari JSA. Vitamin D and attainment of peak bone mass among peripubertal Finnish girls: a 3-y prospective study. Am J Clin Nutr 2002; 76: 1446-1453.

29. Ryan LM, Teach SJ, Singer SA, et al. Bone mineral density and vitamin D status among African American children with forearm fractures. Pediatrics 2012; 130: e553-e560.

30. Jung DW, Park JH, Kim DH, et al. Association between serum ferritin and hemoglobin levels and bone health in Korean adolescents: A nationwide populationbased study. Medicine (Baltimore) 2017; 96: e9403.

31. Rubeor A, Goojha C, Manning J, White J. Does iron supplementation improve performance in irondeficient nonanemic athletes? Sports Health 2018; 10: 400-405. 